\title{
Dramatic changes in electronic structure revealed by fractionally charged nuclei
}

\author{
Aron J. Cohen ${ }^{1}$ and Paula Mori-Sánchez ${ }^{2, a)}$ \\ ${ }^{1}$ Department of Chemistry, Lensfield Rd., University of Cambridge, Cambridge CB2 1EW, United Kingdom \\ ${ }^{2}$ Departamento de Química, Universidad Autónoma de Madrid, 28049 Madrid, Spain
}

(Received 9 August 2013; accepted 13 December 2013; published online 28 January 2014)

\begin{abstract}
Discontinuous changes in the electronic structure upon infinitesimal changes to the Hamiltonian are demonstrated. These are revealed in one and two electron molecular systems by full configuration interaction (FCI) calculations when the realm of the nuclear charge is extended to be fractional. FCI electron densities in these systems show dramatic changes in real space and illustrate the transfer, hopping, and removal of electrons. This is due to the particle nature of electrons seen in stretched systems and is a manifestation of an energy derivative discontinuity at constant number of electrons. Dramatic errors of density functional theory densities are seen in real space as this physics is missing from currently used approximations. The movements of electrons in these simple systems encapsulate those in real physical processes, from chemical reactions to electron transport and pose a great challenge for the development of new electronic structure methods. () 2014 AIP Publishing LLC. [http://dx.doi.org/10.1063/1.4858461]
\end{abstract}

\section{INTRODUCTION}

How electrons move upon a change in the external potential, $v(\mathbf{r})$, is a key question in the understanding of the quantum nature of electrons in matter, given by the Schrödinger equation

$$
\left(\sum_{i}-\frac{1}{2} \nabla_{i}^{2}+v\left(\mathbf{r}_{i}\right)+\sum_{i>j} \frac{1}{r_{i j}}\right) \Psi=E \Psi .
$$

The change in $v(\mathbf{r})$ in processes such as stretching bonds, chemical reactions, and electron transport are great challenges for electronic structure theory. Methods such as Hartree-Fock (HF) and MP2 work well for many properties such as equilibrium structures, where the electronic structure is dominated by a single determinant, however they break down when the basic description of the wavefunction needs more than one determinant. Currently, the only way to tackle this challenge is with multi-reference methods leading to an exact diagonalization of the full Hilbert space with Full Configuration Interaction (FCI), ${ }^{1,2}$ where the limitation is the exponentially scaling size of the space. Density Functional Theory (DFT) attacks the same problem in a fundamentally different manner using the real-space electron density as the fundamental variable, with all the complexity now hidden in the exchangecorrelation functional $E_{x c}[\rho(\mathbf{r})]$. The same functional has to correctly describe all systems, i.e., the result of the functional on many systems is equivalent to many FCI calculations. The simplest example of the different challenge this provides to the electronic structure problem is the incapability to make one functional that describes the energy of both stretched $\mathrm{H}_{2}^{+}$ and stretched $\mathrm{H}_{2} \cdot{ }^{3}$ From a FCI perspective these two systems are trivial as they have one and two electrons, however in DFT it is the use of the same functional that connects them (and in

$\overline{\text { a) Electronic mail: paula.mori@uam.es }}$ fact all other systems) together that poses a distinct challenge. A deeper understanding of the basic behavior of electrons in real space is needed and is the focus of this work.

\section{FRACTIONALLY CHARGED NUCLEI}

A connection between different chemical systems is investigated in this work by taking the normal external potential for a molecule, given by a set of nuclei $\{A\}$ at positions $\mathbf{R}_{A}$ with charge $Z_{A}$,

$$
v(\mathbf{r})=-\sum_{A} \frac{Z_{A}}{\left|\mathbf{r}-\mathbf{R}_{A}\right|},
$$

and simply extending the realm of the charge of the nuclei from the usual integers, $Z_{A} \in \mathbb{Z}$ to now be fractional, $Z_{A} \in \mathbb{R}$. Fractional nuclei have been used previously in different contexts, from finite size scaling ${ }^{9}$ to alchemical changes ${ }^{10,11}$ to inverse-design ${ }^{12,13}$ to Quantum Mechanics/Molecular Mechanics (QM/MM) evaluation of $\mathrm{pKa}^{14}$ to density of atoms. ${ }^{15}$ Here, the idea is used to give a simple and physical controllable change to the Hamiltonian such that, as theoreticians, a fine control over the electronic structure problem is achieved. It should be noted that only the external potential is changed such that we are still solving the Schrödinger equation (Eq. (1)), hence all electronic structure methods should apply. Other physically motivated ideas such as model hamiltonians ${ }^{16}$ like the Hubbard model or Anderson model are different in that they also change the electronelectron interaction and, for example, conventional DFT functionals cannot be directly applied. Although this extension now means that the molecules are fictitious it is key to understand that the electrons are still very real, hence any insights into the understanding of electronic structure are directly applicable to the understanding of real chemical and physical systems. 
(a) Exact

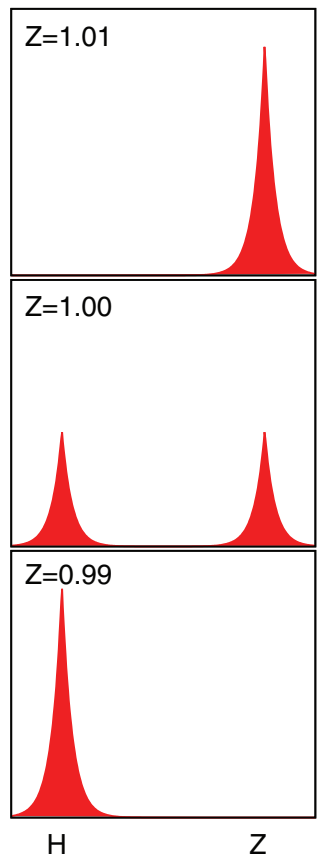

(c) Exact

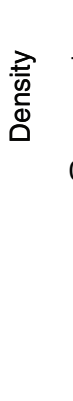

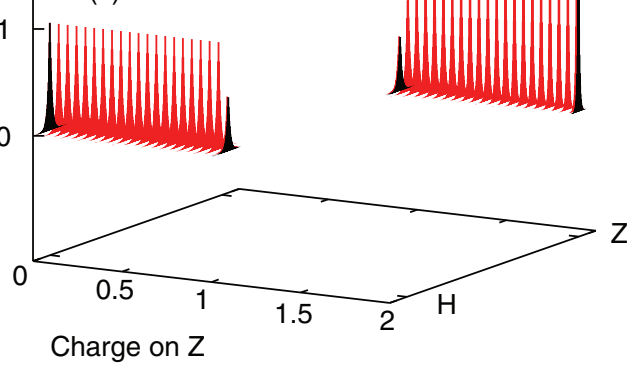

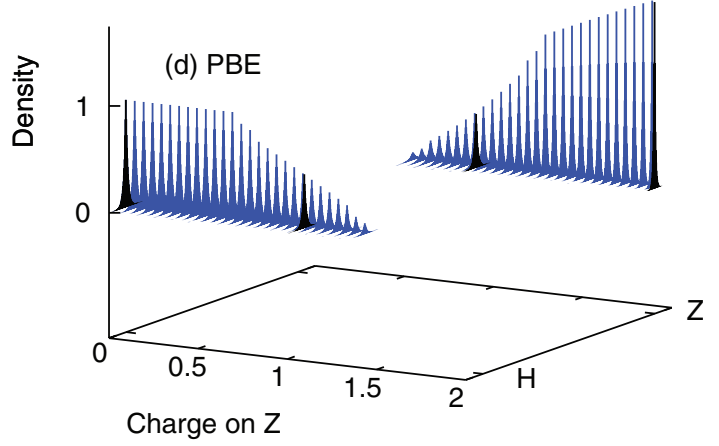

(b) PBE

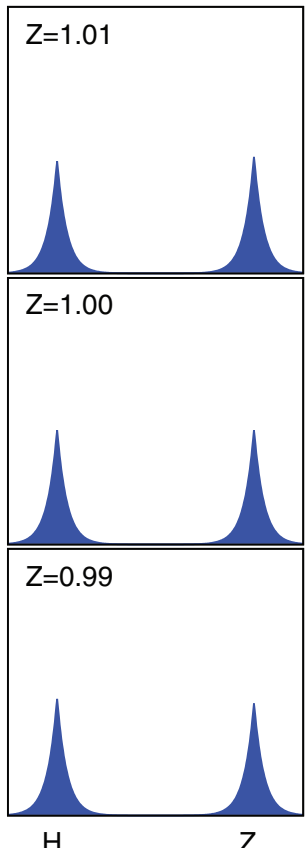

FIG. 1. HZ ${ }^{(1 e)}$ : two protons with one electron, and varying the charge of one of the protons, $0 \leq Z \leq 2$. The density is shown for (a) exact and (b) PBE for three charges on the $Z$ atom $(0.99,1.00,1.01)$. The exact behavior shows very discontinuous behavior at $Z=1$ that a functional such as PBE fails to capture due to delocalization error. In (c) and (d) the same is illustrated but showing the whole range $0 \leq Z \leq 2$, the curves in black correspond to the density for the integer points: $\mathrm{H}$ atom, $\mathrm{H}_{2}^{+}$, and $\mathrm{HHe}^{2+}$.

The exact behavior of electrons in some interesting but simple model systems is now investigated using these fractional nuclear charges. Full configuration interaction calculations ${ }^{17,18}$ are carried out and the behavior of the electron density $\rho(\mathbf{r})$ is examined. This reveals basic intricacies of electronic structure and fundamental behavior of electrons that can be seen in real space. This approach is applied to several simple examples with one and two electrons that are able to reveal fundamental challenges of describing the intricate nature of the quantum mechanical behavior of electrons. Remarkably, the visualization of dramatic changes in the density associated with the integer nature of electrons emerge and are possible to visualize in simple systems.

\section{MOLECULES WITH FRACTIONAL NUCLEI AND ONE ELECTRON}

First consider the $\mathrm{H}_{2}^{+}$molecule with two protons and one electron. From a wavefunction perspective this molecule is trivial as it only has one electron and Hartree-Fock gives the exact solution. However, it still offers challenging behavior that can be illustrated by the failure of non-wavefunction methods such as DFT with local density approximation (LDA) or generalized gradient approximation (GGA) functionals, which upon stretching give a massive error in the energy of around $60 \mathrm{kcal} / \mathrm{mol}$. This is the classic problem of self-interaction ${ }^{19,20}$ or delocalization error. ${ }^{21}$ All semi-local functionals have a qualitative failure in the energy at infin- ity, however, the density is not qualitatively wrong, as it is constrained by symmetry to correctly give half an electron on each end. In Fig. 1 we use fractional nuclei to turn this error in the energy into an error in the density. The charge on one of the protons (now called $\mathrm{Z}$ ) is changed and allowed to be non-integer. The number of electrons is always fixed, constant at 1 , hence we denote this molecule $\mathrm{HZ}^{\{1 e\}}$. The exact behavior from FCI is very simple and clear at infinite separation of $H$ and $Z$. For any $Z<1$ all the electron is on the $H$ (with corresponding energy $-\frac{1}{2}$ ) and for $Z>1$ all the electron is on the $Z$ (with corresponding energy $-\frac{Z^{2}}{2}$ ). For the point at $Z=1$ (corresponding to $\mathrm{H}_{2}^{+}$) the electron can be found half on the $\mathrm{H}$ and half on the $\mathrm{Z}$. Thus, the exact behavior of the energy and electron density show a derivative discontinuity with respect to $Z$ at $Z=1$, clearly exhibiting the integer nature of electrons. It is found that an infinitesimal change in the Hamiltonian produces a dramatic change of the electron density, this is perhaps related to the symmetry breaking seen in $\mathrm{H}_{2}^{+}$in the large dimensional limit. ${ }^{22}$ This can be compared to the performance of the Perdew-Burke-Ernzerhof (PBE) functional, ${ }^{4}$ though the same would be seen for any semi-local LDA, GGA, or meta-GGA functional. PBE clearly misses the discontinuity at $Z=1$ and favors a smooth charge transfer that leads to an over delocalized electron density that is on both $\mathrm{H}$ and $\mathrm{Z}$. The density for non-integer $\mathrm{Z}$ reveals the delocalization error in a very visual manner. Thus, the error for the energy of infinitely stretched $\mathrm{H}_{2}^{+}$is turned into an explicit error for the density in $\mathrm{HZ}^{\{1 e\}}$. It is the first time that the delocalization error is visualized in such a clear manner in a real space picture of a one-electron system. 
TABLE I. Performance of approximate DFT functionals for one electron systems: the atomization energy of $\mathrm{H}_{2}^{+}$and the density of $\mathrm{HZ}^{\{1 e\}}$ as $Z$ is varied as shown by the values (rounded to 2 decimal places) at which functionals begin $\left(Z_{<}\right)$and end $\left(Z_{>}\right)$transferring an electron. Larger errors are seen for GGA functionals $\left(\mathrm{PBE}^{4}\right)$ than for hybrids $\left(\mathrm{PBE}^{5}\right)$ and range-separated hybrids (CAM-B3LYP, ${ }^{6}$ LC-BLYP, ${ }^{7}$ and $\mathrm{rCAM}-\mathrm{B} 3 \mathrm{LYP}^{8}{ }^{8}$.

\begin{tabular}{lccc}
\hline \hline Functional & $\Delta E\left(\mathrm{H}_{2}^{+}\right)$ & $Z_{<}$ & $Z_{>}$ \\
\hline LDA & 56.0 & 0.66 & 1.33 \\
PBE & 60.5 & 0.65 & 1.34 \\
PBE0 & 44.8 & 0.74 & 1.26 \\
CAM-B3LYP & 32.3 & 0.83 & 1.19 \\
LC-BLYP & 25.1 & 0.86 & 1.14 \\
rCAM-B3LYP & 16.0 & 0.91 & 1.10 \\
HF/SIC-LDA & 0.0 & 1.00 & 1.00 \\
\hline \hline
\end{tabular}

This simple exercise is highly illustrative of the complexity of electronic structure that occurs even in one-electron systems. There have been many previous papers on $\mathrm{H}_{2}^{+}$, including many which have highlighted qualitative failures of the energy of DFT functionals, but none of them have focused on such an error in the density. It should be noted that the corresponding "chemical" change (with no fractional nuclei), going from $\mathrm{H}_{2}^{+} \rightarrow \mathrm{HHe}^{2+}$, does not illustrate this failure as the error of functionals is dwarfed by the difference in energy of an electron being on the two different atoms. From a density functional perspective the qualitative failure is a consequence of the delocalization error that can be easily seen in real space. From a chemical point of view, this shows the particle nature of the electrons. This key as- pect is missing from many currently used approximations in DFT.

In Table I we see the performance of approximate functionals for $\mathrm{HZ}^{\{1 e\}}$ calculations at a bond length of $10 \AA$ varying the nuclear charge of one of the protons. The well known error in the energy of the atomization energy of $\mathrm{H}_{2}^{+}$is shown in the first column, and the second and third columns show critical values of $Z$ : for $Z$ values less than $Z_{<}$all the electron will be found on the $\mathrm{H}$ and for all values above $Z_{>}$all the electron is found on the Z. Mulliken populations ${ }^{23}$ are used, other partitioning schemes (e.g., Hirshfeld ${ }^{24}$ ) give practically identical results and agree to the number of decimal places given in Table I.

Fractional nuclei are a very simple way to control and vary the external potential that allow us to see the electrons move. It is of no concern that these molecules are fictitious as it is only a tool to enable us to see what happens to electrons in more complex systems. For example, consider a very simple QM/MM example where an $\mathrm{H}_{2}^{+}$molecule is next to a rotating molecular mechanics water molecule. This is illustrated in Fig. 2 where we rotate a point charge model of a water molecule next to a $10 \AA$ separated $\mathrm{H}_{2}^{+}$molecule. In exactly the same way as in Fig. 1 the electron is transferred as the dipole moment passes through $90^{\circ}$. From the point of view of the performance of approximate functionals in DFT this is a classic case of self-interaction and the performance of approximate functionals is as expected, a GGA functional such as PBE suffers from delocalization error and functionals such as Hartree-Fock or self-interaction corrected methods (e.g., SIC-LDA ${ }^{19}$ ) improve, in fact they are even exact for

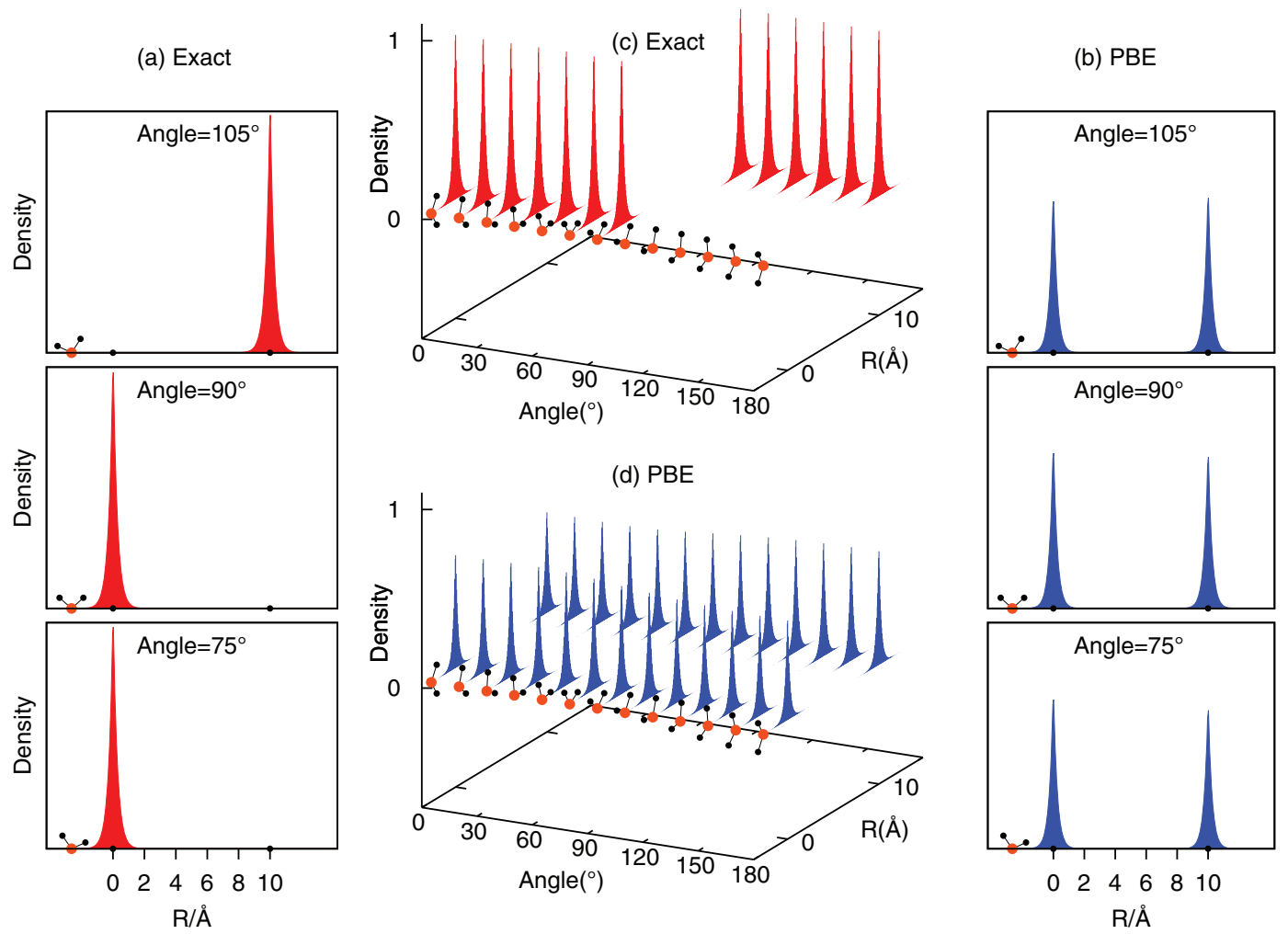

FIG. 2. $\mathrm{H}_{2}^{+}$at $10 \AA$ separation with one of the nuclei $3 \AA$ from a rotating classical dipole moment in the form of a TIP/3P water molecule. The density is shown for (a) exact and (b) PBE as the dipole moment crosses a $90^{\circ}$ angle to the $\mathrm{H}-\mathrm{H}$ bond, the exact behavior shows a discontinuous jump of the electron from one hydrogen to the other that a functional such as PBE fails to capture due to delocalization error. In (c) and (d) the same is illustrated but showing the whole range. 

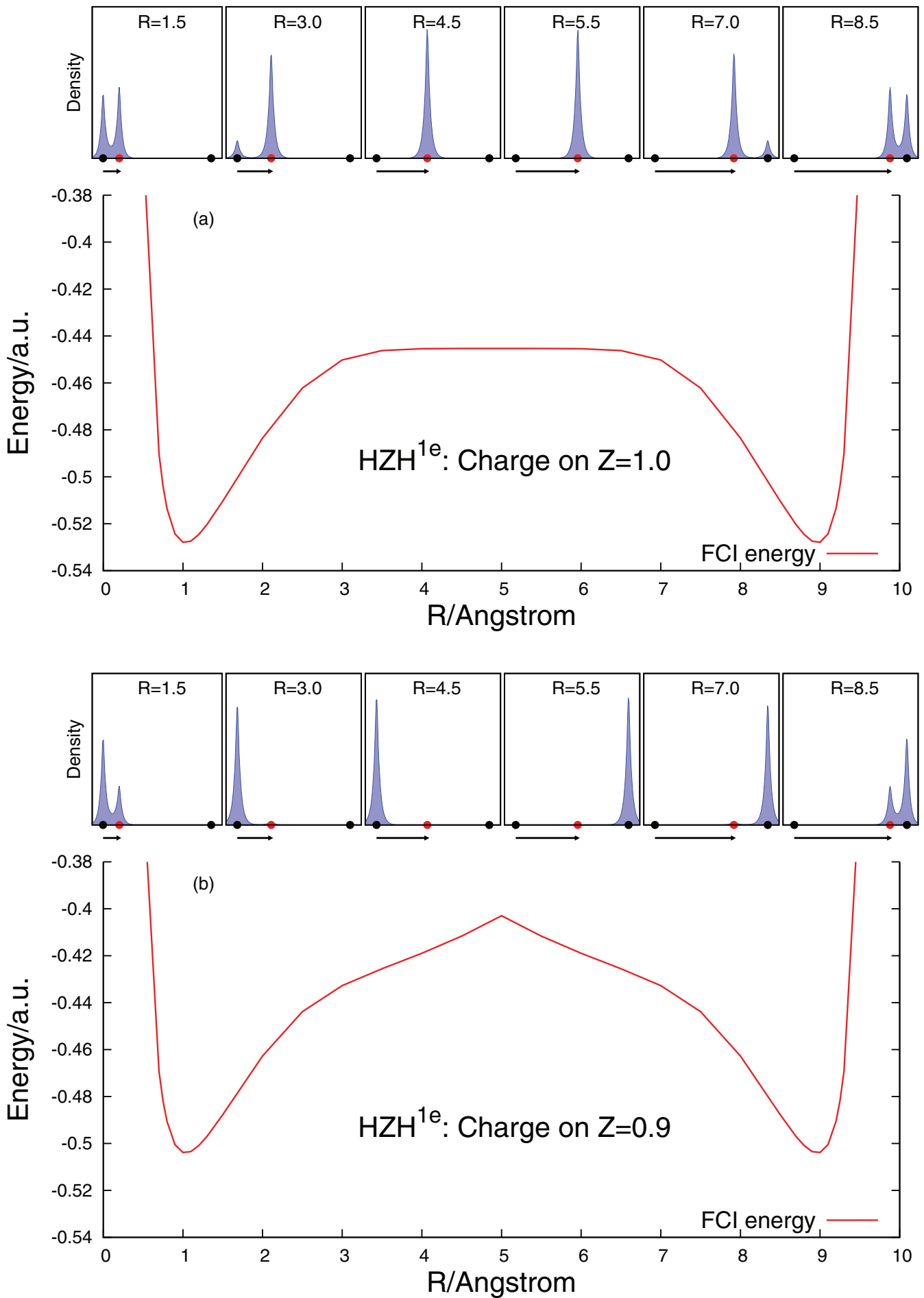

FIG. 3. (a) The energy and density of $\mathrm{HHH}^{2+}$ as the central atom is moved at a distance R between two $\mathrm{H}$ atoms at 0 and $10 \AA$ A The inset pictures show the density for several values of R. The electron is carried across on the central proton as it moves. (b) The same as (a) but now HZH ${ }^{1 e}$ with a fractionally charged nucleus $\mathrm{Z}=0.9$ on the central atom. The inset pictures of the density show the electron hopping between the two stationary protons as the $\mathrm{Z}$ proton moves from the left to the right.

this one-electron problem. It is clear that the same behavior will be seen in complex physics and chemistry such as the electron transfer in systems such as $\mathrm{Fe}^{2+}(\mathrm{aq})+\mathrm{Fe}^{3+}(\mathrm{aq}),{ }^{25}$ the reorganization of the solvent is key to the transfer of electrons.

Next consider the simplest possible chemical reaction with three protons and one electron, $\mathrm{H}_{2}^{+}+\mathrm{H}^{+} \rightarrow \mathrm{H}^{+}+\mathrm{H}_{2}^{+}$.
For simplicity, a linear geometry is taken, with two protons fixed $10 \AA$ apart and another proton moving between them. A one-dimensional coordinate, $\mathrm{R}$ (distance to the left proton), describes the reaction. For $\mathrm{R}=1.0 \AA$ the electron is near the left proton and for $\mathrm{R}=9.0 \AA \mathrm{A}$ the electron is near the right proton. Therefore, as the central proton moves from the left to the right the electron will be transferred as well. 

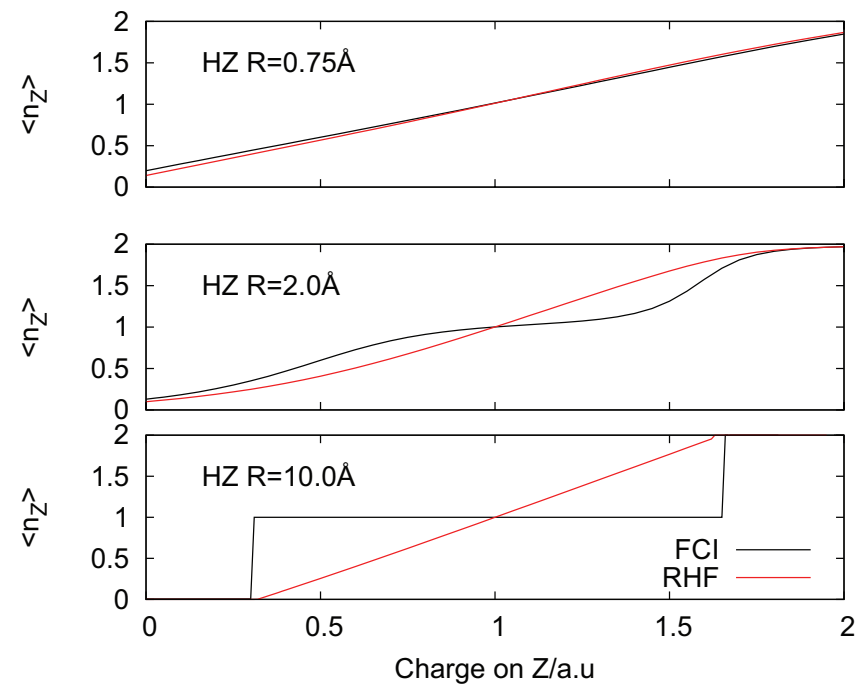

FIG. 4. HZ ${ }^{\{2 e\}}$ system: occupation on the $\mathrm{Z}$ atom $\left(\left\langle n_{Z}\right\rangle\right)$ for charge on atom $\mathrm{Z}, 0 \leq Z \leq 2$, for three different geometries comparing exact FCI with an approximate restricted Hartree-Fock (RHF) method.

We also consider changing the charge on the central proton to be fractional, giving a reaction $\mathrm{HZ}^{\{1 e\}}+\mathrm{H}^{+} \rightarrow \mathrm{H}^{+}$ $+\mathrm{ZH}^{\{1 e\}}$. Fig. 3(a) illustrates the hydrogen atom transfer reaction with a charge $Z=1.0$. The density plots show how the electron is carried on the central proton as it moves from left to right. However, with a charge $\mathrm{Z}=0.9$ on the central proton the reaction exhibits a different mechanism (Fig. 3(b)) proton transfer followed by electron transfer. Here, as the central proton moves $1 \lesssim \mathrm{R}<5$ the electron stays on the left $\mathrm{H}$ atom. This can easily be understood from the previous example, $\mathrm{HZ}^{\{1 e\}}$, where the stretching leaves the electron on the H. However, for the same reason, when $\mathrm{R}>5$ the electron is on the right $\mathrm{H}$ atom. Therefore, there is an electron hopping as $\mathrm{R}$ goes through the midpoint, $\mathrm{R}=5$. This is a very striking example of a conical intersection again showing how a very small change to the system leads to markedly different behavior of the electrons. Electronic structure methods must be able to describe all of these mechanisms correctly to provide a full understanding of chemical reactions and electron transfer processes. The performance of GGA methods for these reactions is disastrous due to delocalization error, with the electron spread over all three centers and a corresponding unphysical drop in energy.

\section{MOLECULES WITH FRACTIONAL NUCLEI AND TWO ELECTRONS}

Let us now consider closed-shell systems with two electrons. We first study the $\mathrm{HZ}^{\{2 e\}}$ system as the nuclear charge on the $\mathrm{Z}$ is varied from 0 to 2 . This connects smoothly from $\mathrm{H}^{-}$to $\mathrm{H}_{2}$, and to $\mathrm{HHe}^{+}$. At stretched geometries these three integer cases have different occupations on the $Z$ atom, with 0,1 , and 2 electrons, respectively. The occupation of the $Z$ atom $\left\langle n_{Z}\right\rangle$ (calculated by a simple Mulliken atomic population) is plotted in Fig. 4 as a function of the charge on the nucleus $\mathrm{Z}$ at three different geometries: around equilibrium $(0.75 \AA)$, moderately stretched $(2 \AA)$, and further stretched $(10 \AA)$. The transfer of electrons as a function of $Z$ is dif- ferent in the three distance regimes. At short bond lengths the transfer happens smoothly however at $10 \AA$ the behavior is very discontinuous. The first electron transfers around $Z=0.29$; this value decreases slightly at longer bond lengths where with the same large diffuse basis set at $1000 \AA$ we find the electron transfer at $Z=0.24$. This agrees well with the infinite limit where the transfer happens when the energy of one electron on the $\mathrm{Z}\left(-Z^{2} / 2\right)$ equals the negative of the electron affinity of the $\mathrm{H}$ atom $(-0.0277$ Hartrees), i.e., $Z=0.235$. A second electron is transferred from the $\mathrm{H}$ to the $\mathrm{Z}$ when the negative of the electron affinity of the $\mathrm{Z}$ atom is equal to the energy of the $\mathrm{H}$ atom ( -0.5 Hartrees), this occurs discontinuously at $Z=1.67$. Restricted Hartree-Fock (RHF) is able to describe the smooth transfer that occurs at shorter distances but fails qualitatively in the stretched limits to give any discontinuous behavior associated with electron hopping. The same is true for any DFT method that misses the derivative discontinuity. It does not give a step like behavior in the occupation $\left\langle n_{Z}\right\rangle$ and hence it does not correctly describe the integer nature of electrons in such electron transfer processes. It is a key point of this paper and illustration of the use of fractional nuclei that this movement of electrons encapsulated in $\mathrm{HZ}^{\{2 e\}}$ is directly equivalent to that seen in the classic Anderson model of electron transport, ${ }^{26-28}$ for example, compare Fig. 4 of this work with Fig. 2 of Ref. 26. However, HZ ${ }^{\{2 e\}}$ offers a much simpler connection to usual chemical concepts (real electrons and nuclei in three-dimensional space) such that, for example, DFT approximations can easily be applied and tested. This test of the density of stretched $\mathrm{HZ}^{\{2 e\}}$ is a very challenging problem for approximate DFT functionals and clearly identifies the challenge of the derivative discontinuity that can be seen qualitatively in a self-consistent density.

In most physical situations, a small perturbation of the Hamiltonian produces a small change in the electronic structure; however, these model systems also show interesting phenomena where a small perturbation, for example, changing the nuclear charge around $Z=1$ in the case of $\mathrm{HZ}^{\{1 e\}}$ or a movement in the geometry around the midpoint of $\mathrm{HZH}^{\{1 e\}}$, leads to dramatic changes in the electron density. In these situations the consideration of the linear density response function, $\chi\left(\mathbf{r}, \mathbf{r}^{\prime}\right)=\left(\frac{\delta \rho(\mathbf{r})}{\delta v\left(\mathbf{r}^{\prime}\right)}\right)_{N}$, is a challenge for approximate theories. ${ }^{29}$ Of course any form such as LDA or GGA does not reproduce the correct underlying electronic structure or linear response, $\chi$. Another related second order response property is the Fukui function for electron removal, $f^{-}(r)$ $=\left(\frac{\partial \rho(\mathbf{r})}{\partial N_{-}}\right)_{v(\mathbf{r})}=\rho^{N}(\mathbf{r})-\rho^{N-1}(\mathbf{r})$, that for the case of $\mathrm{HZ}^{\{2 e\}}$ connects together the densities of $\mathrm{HZ}^{\{2 e\}}$ and $\mathrm{HZ}^{\{1 e\}}$. The $f^{-}(\mathbf{r})$ around $\mathrm{Z}=1$ shows interesting and challenging behavior as even though the density (and hence orbitals) for $\mathrm{HZ}^{\{2 e\}}$ have a smooth behavior with no interesting features around $\mathrm{Z}=1$, the Fukui function shows up a clear discontinuous behavior due to the discontinuity in the density in $\mathrm{HZ}^{\{1 e\}}$, which is not captured by the orbitals. Again the usual derivative expression for the Fukui function ${ }^{30}$ fails completely with currently used functionals, as they are missing the derivative discontinuity.

Finally, we look at the electronic structure in $\mathrm{H}_{2}$ like molecules and change the charge on both of the nuclei: $\mathrm{Z}_{2}^{\{2 e\}}$. Infinitely stretched $\mathrm{Z}_{2}^{\{2 e\}}$ binds two electrons, one on each 
nucleus with a total energy of $-Z^{2} E_{h}$, for all values $Z>0$. Let us now consider the opposite extreme with zero separation, the united atom, that is a single nucleus with charge $2 Z$ that holds 2 electrons. For example, for two $Z=1$ protons (i.e., $\mathrm{H}_{2}$ ) the corresponding united atom is a $\mathrm{He}$ atom, whereas for $Z=0.5$ protons a united atom of $\mathrm{H}^{-}$is obtained. Note that the second electron in $\mathrm{He}$ is bound by $-0.903 E_{h}$ (the electron affinity of $\mathrm{He}^{+}$) whereas the second electron in $\mathrm{H}^{-}$is only just bound, as the electron affinity of the $\mathrm{H}$ atom is now only $-0.028 E_{h}$. Hence, if the nuclear charge on the protons is further reduced to 0.45 , the united atom will have a charge of 0.9 . It is unable to bind two electrons as Kais and co-workers, using finite size scaling, showed that the critical value for the nuclear charge atom to bind two electrons is given by $\mathrm{Z}=0.911 .^{31}$ Therefore, at some point in between $\infty$ and 0 , for $Z=0.45$, the $Z_{2}^{\{2 e\}}$ system undergoes a transition from being able to bind two electrons to only being able to bind one electron. This concept is illustrated in Fig. 5 which contains the binding curves of both $\mathrm{Z}_{2}^{\{1 e\}}$ and $\mathrm{Z}_{2}^{\{2 e\}}$ with a nuclear charge of 0.45 from FCI calculations in a large diffuse basis set. For large bond lengths the energy with two electrons is much lower than the energy with one electron. However, as the bond length is decreased the second electron becomes more weakly bound until at around $2 \AA$ the molecule only binds one electron. At this point the two electron system has an ionization energy that is the same as the electron affinity, $I=A=0$, and the unbound electron is delocalized over all space. This is quite intriguing behavior as a function of geometry, the molecule goes from being able to bind two electrons at long distances to only being able to bind one electron at shorter distances. No similar physics is seen in the corresponding integer nuclear charge system, $\mathrm{H}_{2}$. In future work, we hope to study the connection of this behavior in $\mathrm{Z}_{2}^{\{2 e\}}$ to metal-insulator transitions in the solid state. It should also be noted that density functional approximations such as PBE completely fail to describe this behavior as they have an incorrect $\mathrm{Z}_{2}^{\{1 e\}}$ curve (delocalization

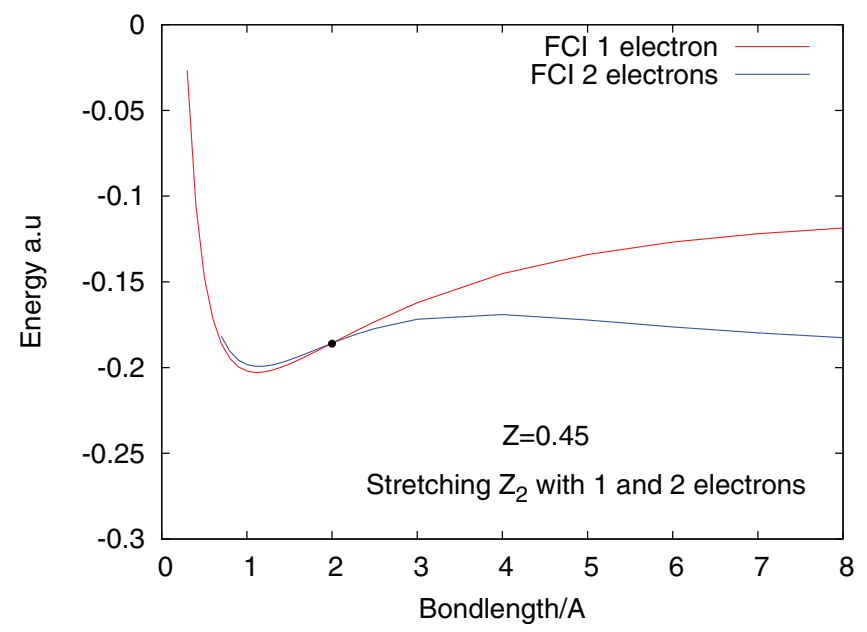

FIG. 5. The full CI energy with one and two electrons of the $Z_{2}$ molecule with charge of 0.45 on both the protons calculated for different bond lengths. There is interesting behavior at an internuclear distance around $2 \AA$, characterized by $I=A=0$ and an unbound electron. error $^{21}$ ), an incorrect $Z_{2}^{\{2 e\}}$ curve (static correlation error ${ }^{32}$ ), and an incorrect energetic preference for $\mathrm{Z}_{2}$ with fractional numbers of electrons (the failure for the flat-plane ${ }^{33}$ ).

\section{CONCLUSIONS}

The foray into the theoretical world of fractionally charged nuclei allows us to directly visualize fundamental complexities of electronic structure in real space. The integer nature of electrons is critical in processes such as electron transfer or conductance and is seen in the $\mathrm{HZ}^{\{1 e\}}$ and $\mathrm{HZ}^{\{2 e\}}$ molecules. $\mathrm{HZ}^{\{1 e\}}$ shows the particle behavior of a single electron. This is not described by approximate functionals in DFT due to an inherent bias towards fractional electrons leading to a delocalization error that can be clearly seen in real space. It should be noted, however, that $\mathrm{HZ}^{\{1 e\}}$ does not capture the derivative discontinuity as it only has a single electron and, for example, $E_{x c}[\rho]=-J[\rho]$ (a smooth differentiable functional with no derivative discontinuity) is exact. A clear picture of the derivative discontinuity is given by the density of stretched $\mathrm{HZ}^{\{2 e\}}$ and shows a useful connection to previous work on the Anderson model. ${ }^{26}$ This illustrates the integer nature of two electrons that is very challenging to describe, for example, an orbital functional such as RHF completely fails. Electron hopping and a conical intersection can be seen in the chemical reaction of the $\mathrm{HZH}^{\{1 e\}}$ system. The $\mathrm{Z}_{2}^{\{2 e\}}$ molecule with $Z=0.45$ shows a geometry dependent transition from binding two electrons to only binding one electron with an electron unbound from the nuclei, characterized by $I=A$ $=0$. The physics encapsulated in the behavior of electrons in all these examples is at the heart of processes from electron transport to chemical reactions and the insulating to metallic transition in materials, which we hope to study more in future work. It is only the use of fractional nuclei that reveals the full complexity of the electronic structure offering a massive challenge for approximate density and wave-function based methods.

\section{ACKNOWLEDGMENTS}

We thank the Royal Society and Ramón y Cajal for funding. P.M.S. also acknowledges Grant No. FIS2009-12721 from the Spanish Ministry of Science and Innovation. We dedicate this work to the memory of Professor N. C. Handy.

${ }^{1}$ P. J. Knowles and N. C. Handy, Chem. Phys. Lett. 111, 315 (1984).

${ }^{2}$ G. H. Booth, A. J. W. Thom, and A. Alavi, J. Chem. Phys. 131, 054106 (2009).

${ }^{3}$ A. J. Cohen, P. Mori-Sánchez, and W. T. Yang, Chem. Rev. 112, 289 (2012).

${ }^{4}$ J. P. Perdew, K. Burke, and M. Ernzerhof, Phys. Rev. Lett. 77, 3865 (1996).

${ }^{5}$ C. Adamo and V. Barone, J. Chem. Phys. 110, 6158 (1999).

${ }^{6}$ T. Yanai, D. P. Tew, and N. C. Handy, Chem. Phys. Lett. 393, 51 (2004).

${ }^{7}$ H. Iikura, T. Tsuneda, T. Yanai, and K. Hirao, J. Chem. Phys. 115, 3540 (2001).

${ }^{8}$ A. J. Cohen, P. Mori-Sanchez, and W. T. Yang, J. Chem. Phys. 126, 191109 (2007).

${ }^{9}$ S. Kais and P. Serra, "Finite-size scaling for atomic and molecular systems," in Advances in Chemical Physics, Vol. 125, edited by I. Prigogine and S. A. Rice (John Wiley \& Sons, Inc., Hoboken, USA, 2003).

${ }^{10}$ O. A. von Lilienfeld and M. E. Tuckerman, J. Chem. Theory Comput. 3, 1083 (2007). 
${ }^{11}$ O. A. von Lilienfeld, Int. J. Quantum Chem. 113, 1676 (2013).

${ }^{12}$ S. Keinan, X. Q. Hu, D. N. Beratan, and W. T. Yang, J. Phys. Chem. A 111, 176 (2007).

${ }^{13}$ X. Q. Hu, D. N. Beratan, and W. T. Yang, J. Chem. Phys. 129, 064102 (2008).

${ }^{14}$ Y. Liu, X. Fan, Y. Jin, X. Q. Hu, and H. Hu, J. Chem. Theory Comput. 9, 4257 (2013).

${ }^{15}$ M. C. Kim, E. Sim, and K. Burke, Phys. Rev. Lett. 111, 073003 (2013).

${ }^{16}$ K. Capelle and V. L. Campo, Jr., Phys. Rep. 528, 91 (2013).

${ }^{17}$ G. Knizia and G. K. L. Chan, Phys. Rev. Lett. 109, 186404 (2012).

${ }^{18}$ G. Knizia and G. K. L. Chan, J. Chem. Theory Comput. 9, 1428 (2013).

${ }^{19}$ J. P. Perdew and A. Zunger, Phys. Rev. B 23, 5048 (1981).

${ }^{20}$ A. Ruzsinszky, J. P. Perdew, G. I. Csonka, O. A. Vydrov, and G. E. Scuseria, J. Chem. Phys. 125, 194112 (2006).

${ }^{21}$ P. Mori-Sánchez, A. J. Cohen, and W. T. Yang, Phys. Rev. Lett. 100, 146401 (2008).
${ }^{22}$ D. D. Frantz and D. R. Herschbach, Chem. Phys. 126, 59 (1988).

${ }^{23}$ R. S. Mulliken, J. Chem. Phys. 23, 1833 (1955).

${ }^{24}$ F. L. Hirshfeld, Theor. Chem. Acta 44, 129 (1977).

${ }^{25}$ R. A. Marcus, J. Chem. Phys. 24, 966 (1956).

${ }^{26}$ J. P. Bergfield, Z. F. Liu, K. Burke, and C. A. Stafford, Phys. Rev. Lett. 108, 066801 (2012)

${ }^{27}$ G. Stefanucci and S. Kurth, Phys. Rev. Lett. 107, 216401 (2011).

${ }^{28}$ P. Tröster, P. Schmitteckert, and F. Evers, Phys. Rev. B 85, 115409 (2012).

${ }^{29}$ M. Hellgren and E. K. U. Gross, Phys. Rev. A 85, 022514 (2012).

${ }^{30}$ W. T. Yang, A. J. Cohen, F. De Proft, and P. Geerlings, J. Chem. Phys. 136, 144110 (2012).

${ }^{31}$ J. P. Neirotti, P. Serra, and S. Kais, J. Chem. Phys. 108, 2765 (1998).

${ }^{32}$ A. J. Cohen, P. Mori-Sánchez, and W. T. Yang, J. Chem. Phys. 129, 121104 (2008).

${ }^{33}$ P. Mori-Sánchez, A. J. Cohen, and W. T. Yang, Phys. Rev. Lett. 102, 066403 (2009) 